\title{
STAATLICHE BETEILIGUNGS- UND VERSTAATLICHUNGSVEREINBARUNGEN MIT DEN AUSLÄNDISCHEN KUPFERBERGBAUGESELLSCHAFTEN IN CHILE UND SAMBIA
}

\author{
Von Wolf RadmanN
}

Staatliche Eingriffe in private Eigentumsrechte hat es in der einen oder anderen Form schon immer gegeben. Anlaß dazu waren sowohl kriegerische Ereignisse als auch soziale, politische und wirtschaftliche Umwälzungen und Reformen. Man denke dabei an die Französische Revolution, an die Wiederaufbaugesetze in den amerikanischen Südstaaten nach dem Sezessionskrieg, an die Konfiskationen in Rußland nach der Revolution von 1917 und später in anderen kommunistischen Ländern oder auch an die Enteignung deutschen Vorkriegsvermögens in den Feindstaaten im Verlaufe des zweiten Weltkrieges. In den letzten Jahren sind Enteignungen privaten Eigentums besonders in Ländern der Dritten Welt gegen in ausländischem Besitz befindliche Unternehmen durchgeführt worden. $\mathrm{Zu}$ all diesen Vorgängen gibt es bereits umfangreiche Literatur und eine Anzahl gerichtlicher Entscheidungen, die sich mit den verschiedenen rechtlichen Gesichtspunkten befassen, die dabei eine Rolle spielen. Dagegen fehlt es noch an Falldarstellungen der bedeutenden Enteignungsvorgänge der letzten Zeit, aus deren Studium neue Erkenntnisse gewonnen werden könnten, um in Zukunft tiefgreifende Krisen der wirtschaftlichen und politischen Zusammenarbeit mit Ländern der Dritten Welt zu vermeiden, die solche staatlichen Enteignungen regelmäßig verursachen ${ }^{1}$.

Dem Beispiel des kürzlich verstorbenen, ehemaligen Präsidenten von Mexiko, General Lazaro Cardenas, der am 18. März 1938 alle ausländischen OOlgesellschaften im Lande verstaatlichte, dem Beispiel der iranischen Regierung bei ihrem Vorgehen gegen die Anglo-Iranian Oil Company in den Jahren 1951-54, das sich kürzlich in den Verhandlungen mit der Iranian Oil Participants, Ltd., fortsetzte, dem Beispiel der ägyptischen Regierung bei der vorzeitigen Übernahme der Compagnie Universelle du Canal Maritime de Suez im Jahre 1956 und auch dem Beispiel Fidel Castros bei der Verstaatlichung des US-amerikanischen Eigentums in Kuba in den Jahren 1959-60 ist eine ansehnliche Zahl anderer Länder der Dritten Welt mit ähnlichen Verstaatlichungsmaßnahmen gefolgt. Es würde den Rahmen dieses Artikels sprengen, wollte man sie alle beschreiben. Im Laufe der Jahre hat sich in dieser Hinsicht jedoch in wenigstens zwei Wirtschaftszweigen eine geplante Zusammenarbeit von Rohstoff ausführenden Ländern entwikkelt, die sich nicht nur auf die Beeinflussung des Weltmarktes, sondern auch auf das Vorgehen gegenüber ausländischen Förderungsgesellschaften erstreckt. Es handelt sich einmal um Öl exportierende und zum anderen um Kupfer exportierende Länder. Davon werden nachfolgend die Verstaatlichungsverfahren in Chile und Sambia beschrieben, welche von den Regierungen des Präsidenten Eduardo Frei und des Präsidenten Kenneth Kaunda gegen die in ihren Ländern

\footnotetext{
1 Schwarzenberger, Foreign Investments and International Law, Stevens \& Son, London, 1969, S. 65; Nehrt, The Political Climate for Private Foreign Investment (North Africa), Praeger Publishers, 1970; Mikesell u. a., Foreign Investment in Petroleum and Mineral Industries, The Johns Hopkins Press, 1971.
} 
ansässigen ausländischen Kupfergrubengesellschaften bisher durchgeführt worden sind. Dabei richtet sich das Interesse hier im wesentlichen auf den Inhalt der zwischen den jeweiligen Regierungen und den ausländischen Gesellschaften getroffenen Vereinbarungen und auf die damit in Zusammenhang stehende $\mathbf{z w i -}$ schenstaatliche Zusammenarbeit.

\section{Zwischenstaatliche Zusammenarbeit Kupfer exportierender Länder}

Im November 1966 stattete Präsident Kaunda, der im Jahre 1965 das Amt des Präsidenten von Sambia übernommen hatte, dem Präsidenten Frei in Santiago de Chile einen Besuch ab. Es war der erste offizielle Besuch, den Präsident Kaunda einem ausländischen Staatsoberhaupt in seiner neuen Funktion machte. Bei dieser Gelegenheit beschlossen beide Staatsmänner, möglichst bald eine Konferenz der bedeutendsten Kupfer exportierenden Länder abzuhalten. Diese Konferenz fand in der ersten Juniwoche 1967 in Lusaka statt, wo kurz vorher die blockfreien Staaten zusammengekommen waren. Die Vertreter der Regierungen Sambias, Chiles, Perus und des Kongo (Kinshasa), denen sich Beobachter aus Uganda und Botswana angeschlossen hatten, kamen überein, eine Organisation der Kupfer exportierenden Länder ins Leben zu rufen, den „Zwischenstaatlichen Rat der Kupfer exportierenden Länder" - C.I.P.E.C. - , dessen Satzung auf der nachfolgenden Tagung in Paris Ende 1967 ausgearbeitet und dort im Mai 1968 unterzeichnet wurde 2 .

Die Präambel der Satzung lautet ${ }^{3}$ :

„... erkennen die außerordentliche Bedeutung von Kupfer für die Wirtschaft ihrer Länder;

sind sich der Notwendigkeit bewußt, übermäßige Schwankungen der Kupferpreise zu verhindern und einen fairen Preis sowohl für Lieferanten als auch für die Verbraucher in Kriegs- und Friedenszeiten aufrechtzuerhalten;

nehmen an, daß die Tendenz zu Preisschwankungen zum Schaden von Lieferanten und Verbrauchern anhalten wird;

glauben, daß diese Situation durch engere Zusammenarbeit und konzertierte Aktion der Kupfer exportierenden Länder verbessert werden kann;

sind sich der Bedeutung der Ausführung der Beschlüsse der zwischenstaatlichen Kupferkonferenz von Lusaka ... bewußt;

und haben folgendes beschlossen..."

Artikel 2 der Satzung befaßt sich mit den Aufgaben der C.I.P.E.C.:

a) Maßnahmen $\mathrm{zu}$ koordinieren, die darauf abzielen, durch Ausdehnung der Industrie, dynamisches und kontinuierliches Wachstum des Realeinkommens aus dem Export von Kupfer zu erreichen und eine realistische Vorhersage solchen Einkommens sicherzustellen;

2 Gueronik, Short History of CIPEC, Paris (CIPEC), Sept. 1970; Standard Bank Review, 1967, Highlights, S. 5; ebenda, Mai 1967, S. 9; ebenda, Juli 1967, S. 15.

3 Dreisprachige Ausgabe der Satzung, erhältlich beim Generalsekretariat der CIPEC, Paris; deutsche Ubersetzung des Verfassers. 
b) Harmonisierung der Entscheidungen und der Politik der Mitgliedsländer in Fragen, die sich auf Produktion und Absatz von Kupfer beziehen;

c) Bessere und vollständigere Unterrichtung sowie einschlägige Beratung in Fragen der Produktion und des Absatzes von Kupfer für die Mitgliedsstaaten;

d) Allgemein, Verbesserung der Mittel zur wirtschaftlichen und sozialen Entwicklung der Erzeugerländer unter Berücksichtigung der Interessen der Verbraucher.

Die erste Konferenz des Ministerrates fand im November 1969 in Lima statt. Obwohl der Gedanke einer Absprache zwischen Regierungen über Fragen der Produktion und des Absatzes bei Grundstofflieferanten insofern nicht neu ist, als eine Reihe Petroleum exportierender Länder schon in Bagdad im September 1960 mit ähnlichen Zielen die Organisation Petroleum exportierender Länder - O.P.E.C. - ins Leben gerufen hatte, wurde der Tatsache, daß es nun auch im Bereich der Kupferindustrie eine derartige Organisation gibt, erhebliche Bedeutung beigemessen.

Chile und Sambia blieben auch weiterhin federführend für die Entwicklung und Ausgestaltung der Zusammenarbeit der CIPEC-Länder innerhalb ihrer Organisation. Aber auch bilateral hatte sich zwischen beiden Ländern seit dem Staatsbesuch Präsident Kaundas in Chile eine engere Zusammenarbeit entwickelt, die sich schließlich darin äußerte, daß die Verstaatlichung der Kupfergruben in Sambia dem in Chile zuvor angewandten Muster folgte. Schon im Jahre 1967 hatte sich ein Beamter der staatlichen chilenischen CODELCO längere Zeit im Mines Department der sambischen Regierung aufgehalten ${ }^{4}$, und andere Regierungsvertreter Chiles hatten Anfang 1968 offizielle Besprechungen mit der sambischen Regierung, die sich auf die Erfahrungen der Chilenen bei den Verhandlungen mit den amerikanischen Kupfergrubengesellschaften erstreckten ${ }^{5}$.

Bedenken der Privatwirtschaft gegen diese Art zwischenstaatlicher Zusammenarbeit wurden von dem Aufsichtsratsvorsitzenden der Anaconda Corporation, C. Jay Parkinson, in seinem Vortrag vor dem American Metal Market's Second Annual London Forum ${ }^{6}$ kürzlich folgendermaßen zusammengefaßt. Die Kupfer verarbeitenden Industrieländer, so sagte er, würden sich in Anbetracht der in den Lieferländern geschaffenen Unsicherheit nach anderen Kupfervorkommen umsehen, deren Länder politisch zuverlässigere Verhältnisse aufwiesen. Weniger ertragreiche Vorkommen würden vorzeitig ausgeschöpft, um den Absatz des Kupfers aus den CIPEC-Ländern zu erschweren. Das würde zu einer Überproduktion führen und damit zu einem drastischen Absinken der Preise zum Nachteil der CIPEC-Länder. Schließlich sei das Verhalten der CIPEC-Länder auch nicht dazu angetan, ein besonders reizvolles Klima für private Investitionen auf anderen Sektoren zu schaffen.

\footnotetext{
4 Standard Bank Review, Juni 1967, S. 10.

5 Standard Bank Review, März 1968, S. 11.

6 Wortlaut der Ansprache: Broschüre der Anaconda Company, New York.
} 


\section{Beteiligung des Staates an den Kupferbergwerksgesellschaften in Chile}

Chile gehört mit Venezuela zu den teuersten Ländern Lateinamerikas. Seine Wirtschaft war in den letzten Jahren von einer galoppierenden Inflation befallen, und sein Staatshaushalt wies ein erhebliches, sich laufend vergrößerndes Defizit aus. Neben der französischen und der italienischen ist die chilenische kommunistische Partei die bedeutendste außerhalb des Bereichs kommunistischer Länder. Die Kupferförderung Chiles war im Jahre 1969 die drittgrößte der westlichen Welt und machte 75 Prozent der gesamten chilenischen Ausfuhren aus. Bisher lag die Kupferförderung fast ausschließlich in Händen US-amerikanischer Gesellschaften, der Anaconda Company, der Braden Copper Company als Tochtergesellschaft der Kennecott Company und der Cerro Corporation.

Seit Jahren hatten linksgerichtete Kreise auf Verstaatlichung der Kupfergruben gedrungen, weil sie sich dadurch höhere Staatseinnahmen versprachen, die es dann ermöglichen sollten, die in ihren Anfängen mangels ausreichender Mittel steckengebliebene Agrarreform voranzutreiben. So fand Präsident Eduardo Frei bei Úbernahme seines Amtes keine sonderlich leichte Aufgabe vor. Um dem ständig wachsenden Druck auf Verstaatlichung entgegenzuwirken, begann er bald nach Amtsantritt mit den drei US-amerikanischen Bergwerksgesellschaften über seinen "Chilenisierungsplan“ zu verhandeln. Dieser Plan sah eine Beteiligung der chilenischen Regierung in Höhe von 51 Prozent an allen größeren Bergwerksgesellschaften vor. Die Braden Copper Company ging auf diesen Vorschlag ein, während die Anaconda Company es zu einem Kompromiß kommen ließ, der wahrscheinlich von der Vorstellung ausging, daß früher oder später eine Verstaatlichung in vollem Umfange nicht zu umgehen sein würde und bis dahin jeder Verhandlungstag und jeder Vergleich auf der Aktivseite der Bilanz erscheinen würde. Nach Abschluß der Verhandlungen gab Präsident Frei das Ergebnis in einer Fernsehansprache am 21. Dezember 1964 seinem Lande bekannt. Die Abkommen sahen folgendes vor:

Das Vermögen der Braden Copper Company (Kennecott) ${ }^{7}$ wurde in eine neu gegründete Auffanggesellschaft, die Sociedad Minera El Teniente, S.A., eingebracht. Dafür übertrug die Auffanggesellschaft sämtliche Anteile auf die bisherige Braden Copper Company, die dann ihrerseits wiederum 51 Prozent der Anteile auf die hundertprozentig in Händen der chilenischen Regierung liegenden Corporación del Cobre, S.A. (CODELCO) übertrug. Der vereinbarte Kaufpreis von $\$ 80$ Millionen war in Wechseln der CODELCO zu entrichten, die von der chilenischen Regierung verbürgt wurden. Das Abkommen sollte bis zum 13. April 1967 abgewickelt werden. Für die Weiterführung der Grubenbetriebe durch die alte Braden Copper Company wurde ein Betriebsführungsvertrag abgeschlossen. Der Vertragskomplex enthielt auch ein Entwicklungsabkommen für den Ausbau der Kupferproduktion um 60 Prozent gegenüber der gegenwärtigen Förderungskapazität von $180000 \mathrm{t}$ jährlich. Die Finanzierung dieses Entwicklungsvorhabens wurde auf $\$ 230$ Millionen geschätzt. Für diesen Betrag sollten Kredite von der ExportImport Bank in Washington in Höhe von $\$ 110$. Millionen, der CODELCO in Höhe von $\$ 27$ Mill. und von der Braden Copper Company in Höhe von

7 Jahresbericht der Kennecott Company sowie Aktionärsmitteilungen; die Verträge selbst sind nicht zugänglich. 
\$93 Millionen gewährt werden. Der Kredit der Braden Copper Company war von der U.S. Agency for International Development (AID) unter ihrem Specific Risk Guaranty Program versichert und sollte jeweils in dem Umfange fällig werden, in dem CODELCO die für die Übertragung der 51prozentigen Beteiligung an El Teniente Mining Company hingegebenen Wechsel einlöste.

Die Compañia Minera Andina - Tochtergesellschaft der US-amerikanischen CERRO Corporation - wurde von dem Chilenisierungsplan nicht betroffen ${ }^{8}$, weil sie nach der Definition des Regierungserlasses außerhalb des Bereichs der „großen“ Gesellschaften lag. Außerdem wurde sie erst im Dezember 1966 zur Ausbeutung der Rio-Blanco-Grube in Zentralchile mit einer Beteiligung von CODELCO in Höhe von 30 Prozent gegründet. Die Cerro Corporation hat in diese Gemeinschaftsgründung bisher etwa $\$ 33$ Millionen investiert, die gegen Verlust durch Enteignung bei der U.S. Agency for International Development versichert sind. An dem Finanzaufwand dieses Unternehmens sind ferner die Export-Import Bank in Washington und die Sumitomo Metal Mining Company, Ltd., Tokio, beteiligt.

Der zur gleichen Zeit von der chilenischen Regierung mit Anaconda ${ }^{9}$ abgeschlossene Vertrag folgte nicht dem sogenannten Chilenisierungsplan, sondern war das, was man ein Assoziierungsprogramm nannte. Die Grundzüge dieses Programmes waren engere Zusammenarbeit zwischen Anaconda Company und der chilenischen Regierung durch Schaffung einer größeren Anzahl von neuen Arbeitsplätzen und beschleunigte Entwicklung neuer Kupfervorkommen. Es wurden zwei neue Gemeinschaftsunternehmen gegründet. An dem einen wurde CODELCO mit 25 Prozent beteiligt. Sie sollte die Entwicklung der weniger bedeutenden Exotica-Grube in der Nähe der größeren Chuquicamata-Grube übernehmen. Beginn der Förderoperationen wurde für 1971 vorgesehen; die feierliche Einweihung erfolgte am 2. Juli 1970. An der zweiten Gesellschaft wurde die Regierung mit 49 Prozent und Anaconda mit 51 Prozent beteiligt; ihr Zweck lag darin, nach neuen Kupfervorkommen zu schürfen. Für die Entwicklung und Erschließung jeden neuen Vorkommens sollte dann eine neue Gesellschaft mit gleichen Beteiligungsverhältnissen gegründet werden. Anacondas größte Tochtergesellschaften, die Chile Exploration Company mit der Chuquicamata-Grube und die Andes Copper Mining Company mit den Gruben in Potrerillos und El Salvador, wurden von diesem Abkommen nicht berührt.

Der Gesamtkomplex der Abkommen mit den Kupferbergwerksgesellschaften sah ferner vor, daß die chilenische Regierung größeren Einfluß auf die Geschäftspolitik erhalten sollte. Er enthielt außerdem ein Steuerabkommen für die nächsten zwanzig Jahre sowie allgemeine Richtlinien für das Verhalten der Bergwerksgesellschaften im Gastlande.

Die folgenden Jahre brachten Chile leider nicht die erhoffte Beruhigung der inflationären Bewegung. Schwere Erdbeben im Jahre 1965 und eine lange Dürre in den Jahren 1967-68 verursachten weitere Belastungen der Volkswirtschaft und sorgten dafür, daß die Preise im Lande weiter stiegen. Der Militärputsch in Peru und die nachfolgende schlagartige Wegnahme der Anlagen der International Petroleum Company, einer Tochter der Standard Oil of New Jersey, am 10. Oktober 1968 ermutigte linksgerichtete Kreise in Chile auch hier auf völlige Enteignung der Kupferbergwerksgesellschaften zu drängen.

8 Jahresbericht und Broschüre der Cerro Corporation.

9 Jahresberichte und Aktionärsmitteilungen der Anaconda Company. 
Die Situation der Kupferbergwerksgesellschaften wurde andererseits nachteilig beeinflußt durch die ungewöhnliche Entwicklung der Kupferpreise auf dem Weltmarkt. Lange Zeit hindurch hatte keine nennenswerte Differenz bestanden zwischen dem Kupferpreis an der Londoner Metallbörse und dem sogenannten Produzentenpreis, der unter den größeren Kupfergesellschaften der westlichen Welt abgesprochen wurde und die Grundlage bildete für die Berechnung der Ausfuhrsteuern und für die in den Konzessionsvereinbarungen mit den Regierungen der exportierenden Länder festgelegten Ertragsabgaben. Der Preis des in den USA gewonnenen Kupfers unterlag diesen Abmachungen indes nicht, weil dessen Ausfuhr zu der Zeit aus strategischen Gründen untersagt war und einer besonderen Ausfuhrgenehmigung bedurfte. Im Jahre 1964 begann dieses internationale Preissystem jedoch aus den Fugen zu gehen und brach 1966 praktisch vorübergehend völlig zusammen, als im Dezember des Jahres der Preis an der London Metal Exchange das Doppelte des Produzentenpreises erreichte.

Eine merkliche Beruhigung dieser Preissituation trat in den folgenden Jahren nicht ein, obwohl das Mißverhältnis sowohl durch verschiedene Erhöhungen des Produzentenpreises als auch durch Nachgeben des Börsenpreises an Schärfe verlor. Dafür gibt es verschiedene Gründe. So hat zum Beispiel der Kupferbedarf von 1960 bis 1965 in den USA nur um 23 Prozent, in der übrigen Welt hingegen um 172 Prozent zugenommen. Das wird damit in Zusammenhang gebracht, daß der Lebensstandard in manchen Ländern erheblich schneller gestiegen ist als in den schon auf hohem Niveau lebenden USA und daß eine Reihe selbständig gewordener Staaten eine eigene Kupfer verarbeitende Industrie aufgebaut haben. Auch nahm der Krieg in Vietnam nach 1964 heftigere Formen an, so daß die Rüstungsindustrie mehr Kupfer verbrauchte. In den USA, in Chile und in Sambia fanden längere Streiks in den Bergwerken statt, durch die zum Beispiel die Kupferindustrie in den USA nahezu 90 Prozent für neun Monate stillgelegt war. Schließlich verursachte die einseitige Unabhängigkeitserklärung Südrhodesiens am 1. November 1965 Schwierigkeiten für den Abtransport des sambischen Kupfers, der bis dahin in erster Linie durch Rhodesien erfolgt war. Nicht zuletzt gehören in diesem Zusammenhang auch die Schwierigkeiten zwischen der Regierung des Kongo (Kinshasa) und der Union Minière du Haut Katanga, die letztlich zur Enteignung der Gesellschaft zum 1. Januar 1967 führte.

Bei der Eröffnung des 154. Kongresses am 21. Mai 1969 nahm Präsident Frei nochmals gegen eine vollständige Verstaatlichung der Bergwerksgesellschaften Stellung, ließ jedoch keinen Zweifel daran, daß Chile mehr an den Gewinnen der Gesellschaften beteiligt werden müßte. Gänzliche Verstaatlichung, meinte er, berge ein unnötiges Risiko für Chile, dem die finanziellen Reserven für Entschädigungsleistungen fehlten: „Wir erachten es an der Zeit, daß diejenigen Gesellschaften, die sich bisher abseits gehalten haben, sich nun in die Gegebenheiten fügen ${ }^{10}$."

Kurz darauf begannen erneut hart geführte Verhandlungen mit Anaconda Company ${ }^{11}$. Das Ergebnis gab Präsident Frei seinem Lande am 28. Juni 1969 in einer Fernsehansprache bekannt ${ }^{12}$. Den Tag der Unterzeichnung des Abkommens mit Anaconda, den 26. Juni 1969, erklärte er zum Feiertag der Verstaatlichung der Kupferproduktion.

10 New York Times 22. Mai 1969.

11 Le Monde, 25. 6. 1969.

12 Le Monde, 28. 6. und 1. 7. 1969. 
Die Anaconda Company hatte sich nicht dem Chilenisierungsplan des Präsidenten Frei schlechthin angeschlossen, sondern hatte einen Gegenvorschlag gemacht, der auf eine langfristige Verstaatlichung der gesamten Anlagen in zwei Etappen abzielte und von der Regierung angenommen worden war. Das Abkommen sah im einzelnen vor ${ }^{13}$ :

Mit Wirkung vom 31. Dezember 1969 war das Reinvermögen der beiden Tochtergesellschaften der Anaconda Company, die Chile Exploration Company mit einer Kapitalisierung von $\$ 275480000$ und die Andes Copper Mining Company mit einer Kapitalisierung vor $\$ 66637000$, in zwei neu zu gründende Auffanggesellschaften einzubringen, die Compañia de Cobre Chuquicamata, S.A., und die Compañia de Cobre Salvador, S.A., deren Anteile dafür auf die Altgesellschaften übertragen wurden. In der ersten Stufe, die sofort ausgeführt werden sollte, veräußerten und übereigneten die beiden Altgesellschaften jeweils 51 Prozent der von ihnen gehaltenen Anteile der jeweiligen Auffanggesellschaft an die CODELCO, die ihrerseits dafür Wechsel in Höhe des Gegenwertes herzugeben hatte, die von der regierungseigenen Corporación de Fomento de la Produción (CORFO) verbürgt wurden. Die restlichen 49 Prozent Anteile an den neuen Auffanggesellschaften sollten in der zweiten Stufe auf gleiche Weise von den Auffanggesellschaften auf die CODELCO übertragen werden. Diese zweite Stufe sollte an einem von der chilenischen Regierung zu bestimmenden Zeitpunkt ab 1972, aber nicht später als bis zum 31. Dezember 1981 abgewickelt werden. Sie war daran gebunden, daß zum Abwicklungszeitpunkt alle bis dahin fälligen Wechsel eingelöst waren. Außerdem sollten bei Einleitung der zweiten Stufe sofort 60 Prozent der noch ausstehenden Wechsel fällig werden. Die Wechsel waren in 24 gleichen, halbjährlichen Raten beginnend mit dem 30. Juni 1970 einzulösen und mit 6 Prozent zu verzinsen. Das Abkommen enthielt ferner eine Überpreis-Klausel, die auf die Basisjahre 1968/69 bezogen war und für die Berechnung der an die Regierung zu entrichtenden Abgaben maßgebend sein sollte.

Nach diesen Bedingungen würde die chilenische Regierung in der ersten Abwicklungsstufe über einen Zeitraum von 12 Jahren ungefähr einen Betrag von $\$ 270$ Millionen zu zahlen haben. Die Gesamtzeit für die volle Verstaatlichung würde bei einem Kostenaufwand von schätzungsweise $\$ 400$ Millionen zwischen 19 und 24 Jahren liegen.

Die beiden Altgesellschaften haben außerdem mit den jeweiligen Auffanggesellschaften Betriebsführungsverträge abgeschlossen, aus denen sie für ihre Tätigkeit ein Honorar in Höhe von einem Prozent des Bruttoverdienstes der beiden Auffanggesellschaften erhalten sollen.

13 Jahresberichte der Anaconda Company; Le Monde, 29. 6. 69; die Verträge selbst sind nicht zugänglich. 


\section{Stufe der Verstaatlichung}

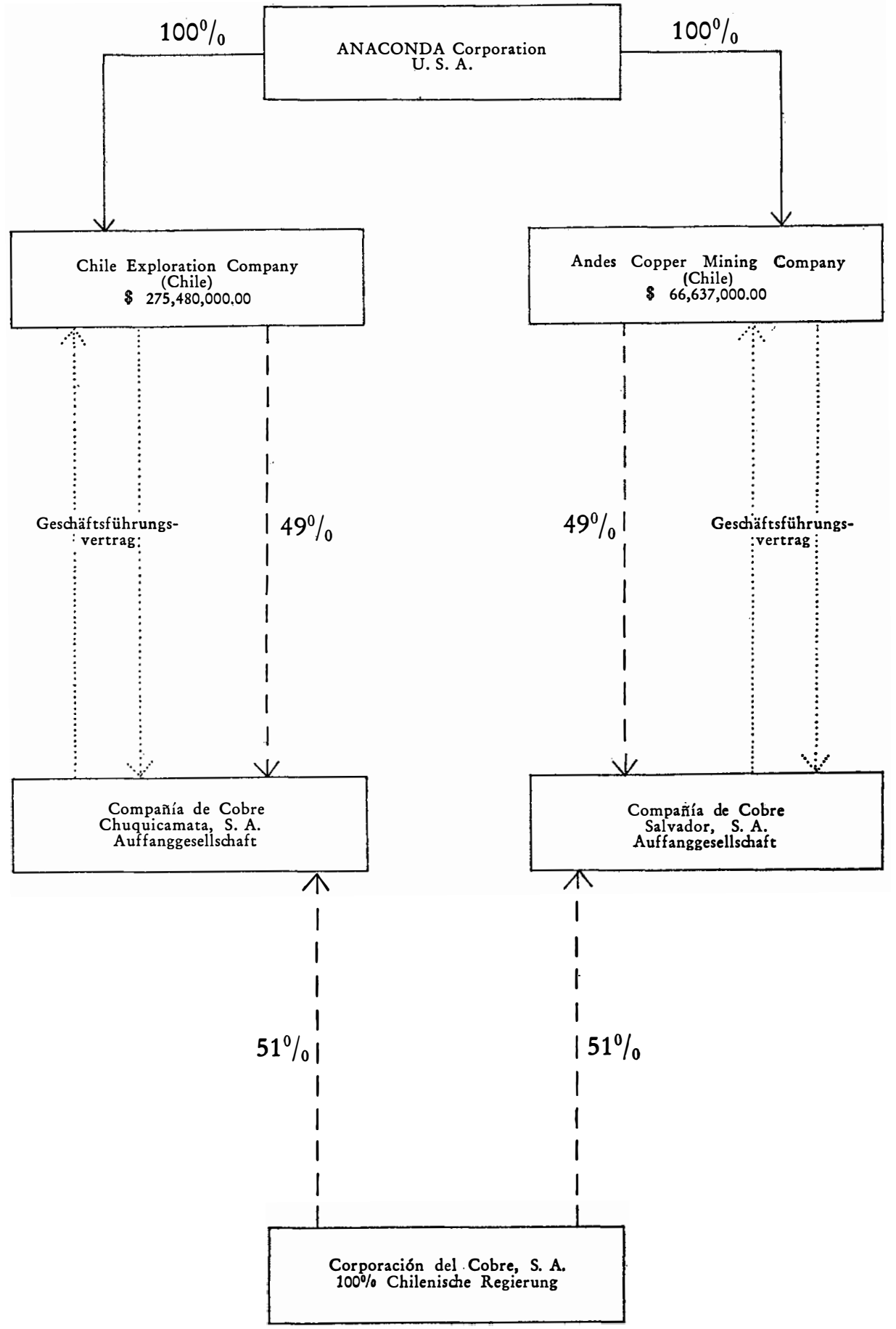




\section{Beteiligung des Staates an den Kupferbergwerksgesellschaften in Sambia}

Sambia erlangte seine Unabhängigkeit im Oktober 1964, etwa zur gleichen Zeit, als Präsident Frei in Chile mit den Bergwerksgesellschaften über sein Chilenisierungsprogramm verhandelte. Die beiden Länder zusammen produzieren etwa die Hälfte des am Weltmarkt gehandelten Kupfers. Sambia ist der drittgrößte Produzent der Welt nach den USA und Rußland und vor Chile und Kanada. Die Kupferexporte machen 90 Prozent des sambischen Exporterlöses aus. Die Kupferproduktion wird von zwei großen Gesellschaften bestritten, der AngloAmerican Corporation of Sambia, Ltd., einer Tochtergesellschaft der Anglo-American Corporation of South Africa, Ltd., und dem Roan Selection Trust, dessen Hauptaktionär die American Metal Climax Corporation in New York ist. Bisher wurden sieben Gruben ausgebeutet, Chililabombwe, Chibuluma, Mujulvia, Nchanga, Nkana und Chambishi.

Zum Unterschied von Chile, das seit der ersten Hälfte des vergangenen Jahrhunderts unabhängig ist und über einen eingefahrenen Regierungs- und Verwaltungsapparat verfügt, mußte Präsident Kaunda das erst in Sambia aufbauen, da dort während der Kolonialzeit die qualifizierten Positionen in Verwaltung und Wirtschaft den Afrikanern nicht zugänglich waren. Sambianisierung ${ }^{14}$ bezog sich deshalb in erster Linie auf die Ersetzung ausländischer Fachkräfte durch einheimisches Personal, während sich Chilenisierung in erster Linie auf ausländisches Kapital bezog. Nach dem von der Regierung einberufenen Brown Commission verfaßten Bericht sollen bis zum Jahre 1972 alle Arbeitsplätze der Schichtführer und Schachtkapitäne mit Sambiern besetzt sein. Ebenfalls bis 1972 soll sich die Zahl der ausländischen Arbeitskräfte in den Bergwerken von 7300 bei Erlangung der Unabhängigkeit auf 3168 verringert haben.

Auch die außenpolitische Situation in Afrika bot in den sechziger Jahren für Sambia mehr Schwierigkeiten, als es für Chile in Lateinamerika der Fall war, so daß es für Kaunda schwerer war, eine neue, eigene Wirtschaftspolitik hinsichtlich der Kupferbergwerksgesellschaften $\mathrm{zu}$ entwickeln und durchzusetzen. Zunächst ging es darum, die Abtransportwege für das im Kupfergürtel geförderte Kupfer zum Weltmarkt sicherzustellen ${ }^{15}$. Wegen der von der Regierung Ian Smith in Südrhodesien am 11. November 1965 ausgesprochenen einseitigen Unabhängigkeitserklärung und der politischen Orientierung des Landes sowohl als auch der portugiesischen Gebiete Mozambique und Angola ging es Präsident Kaunda darum, eine Alternative zum Transport per Bahn durch Südrhodesien nach Laurenço Marques zu finden, auf dem das Kupfer Sambias bisher auf den Weltmarkt gelangt war. Es ging auch darum, nicht auf Verschiffung im Hafen von Beira oder Lobita angewiesen zu sein. Der Weg durch den Kongo nach Matadi ist zudem zu kostspielig und beschwerlich. Deshalb wandte Kaunda sich an Tanzania. Um den Hafen von DarEs-Salaam zu benutzen, zu dem es noch keine Eisenbahnverbindung vom Kupfergürtel aus gibt, wurde im August 1968 ein Gemeinschaftsunternehmen mit Tanzania und italienischen Interessen gegründet, das mit Hilfe eines Lastwagenparks von 450 Fahrzeugen OOl von Dar-Es-Salaam per Straße zum Kupfergürtel bringt und auf dem Rückweg Kupfererz transportiert. Gleichzeitig wurde der Bau einer

14 Etheradge, „Zambianization in the Copper Belt ${ }^{\alpha}$, Optima, Dez. 1969, S. 183 ff., Johannesburg, Südafrika; Vilakazi, "Non-Governmental Agencies and their Role in Development in Africa a Case Study, African Studies Review, Michigan State University, Vol. XIII, Nr. 2, Sept. 1970, S. $169 \mathrm{ff}$.

15 De Lavilleon, „Du Nouveau dans l'Est Africain“, Le Monde 22./23. 8. 1967. 
neuen Autostraße und einer Olleitung in Angriff genommen. Damit war es möglich, die Kupferindustrie von Kohle, die man perEisenbahn aus Südrhodesien beziehen mußte, auf Ơl umzustellen. Ein Abkommen über den Bau einer Eisenbahnanschlußlinie vom Kupfergürtel an das tanzanische Eisenbahnnetz wurde am 18. November 1969 mit Vertretern der Volksrepublik China in Lusaka unterzeichnet.

Parallel zu dieser Entwicklung begannen auch die Vorstellungen Kaundas über die zukünftige Struktur und Verfassung der sambischen Wirtschaft nach außen erkennbar zu werden. Sicherlich unter dem Eindruck der Verstaatlichung der Union Minière du Haut Katanga am 1. Januar 1967 und Präsident Nyereres Erklärung von Arusha im Februar $1967^{16}$ sowie der Veröffentlichung von TANU's Programm des Sozialismus und des Selbstvertrauens unterbreitete Kaunda dem Nationalrat der Vereinigten Nationalen Unabhängigkeitspartei am 26. April 1967 in Mulungushi1 die Grundzüge seiner Wirtschaftspolitik, die für den Bereich der Kupferbergwerksgesellschaften gemischt staatlich-private Initiative oder nach Wahl der Regierung auch ausschließlich staatliche Initiative vorsah. Als erstes Gemeinschaftsunternehmen in der Kupferindustrie wurde im Juni 1968 die „Metal Fabrications of Zambia. Ltd." gegründet ${ }^{18}$. Die Verhandlungen hatten schon im Dezember $1967 \mathrm{zu}$ einer grundsätzlichen Übereinstimmung der Interessenten geführt, wurden aber dadurch verzögert, daß die sambische Regierung nach chilenischem Muster auf einer Mehrheitsbeteiligung von 51 Prozent bestand. Die restlichen 49 Prozent wurden unter folgende Gesellschaften aufgeteilt: Phelps Dodge (USA), Anglo-American Corporation, Roan Selection Trust, Continental Ore, Svenska Metalverk.

Im April 1968 wurde Kaunda in Mulungushi ${ }^{19}$ wesentlich deutlicher und forderte 25 bedeutende Gesellschaften auf, dem Staat eine Mehrheitsbeteiligung von 51 Prozent zur Übernahme anzubieten. Die Kupferindustrie zählte jedoch noch nicht dazu. Präsident Kaunda beklagte sich jedoch, daß sie sich zu wenig um die wirtschaftliche Entwicklung der ländlichen Gebiete kümmere. Es ginge nicht nur darum, die Gruben auszubauen, sondern auch darum, die wirtschaftliche Entwicklung auch in anderen Zweigen zu fördern und neue Arbeitsplätze zu schaffen und den Lebensstandard der Bevölkerung zu heben. Anläßlich der Maifeier in Kitwe bestätigte er dann nochmals, daß die Regierung nicht beabsichtige, die Kupferbergwerke zu verstaatlichen. Sambia benötige das Kapital und das Know-how, das in diesem Bereich konzentriert sei. Sicherheitshalber fuhr der Finanzminister aber in die USA und nach England um die Bedeutung der Erklärung von Mulungushi $\mathrm{zu}$ erläutern.

Im November 1968 wurde eine weitere Gemeinschaftsgesellschaft im Grubenbereich gegründet, die „Metal Marketing Company of Zambia, Ltd.“20, an der der Staat mit 51 Prozent und die Anglo-American Corporation und der Roan Selection 'Trust zusammen mit 49 Prozent beteiligt sind. Der Gesellschaftszweck besteht in der gemeinsamen Erarbeitung einer Marktpolitik, der Revision bestehender Verträge und Festlegung neuer Richtlinien. Der Minister für Land und Bergwerksange-

16 Sunday News, Lusaka, 12. 2. 1967.

17 African Development, London, Jan. 1970, S. 15 ff., "Anatomy of the Zambia Copper Nationalization"; Standard Bank Review, London, July 1970; ebenda, "The Economy in 1969“; Jeune Afrique, Nr. 451, 452, Sept. 1969.

18 Standard Bank Review, March, June, Nov., 1968.

19 Wie Fn 17 oben.

20 Standard Bank Review, London, Dez. 1968, S. 14. 
legenheiten versicherte bei dieser Gelegenheit nochmals, daß die Regierung nicht beabsichtige, die Kupferbergwerksgesellschaften zu verstaatlichen. Auch auf seiner Reise in die USA im Juni 1969 sagte Präsident Kaunda, daß seine Regierung nicht an die Verstaatlichung der Kupferbergwerksgesellschaften denke.

Anläßlich des Parteitages in der Matero Hall in Lusaka am 11. August 1969 sagte Präsident Kaunda schließlich den dort versammelten mehr als 300 Parteiführern, daß die Regierung beschlossen habe, die Kupferbergwerksgesellschaften aufzufordern, dem Staat eine Beteiligung von 51 Prozent anzubieten. Außerdem sollten alle Eigentumsrechte an Bodenschätzen auf den Staat zurückfallen, auch die gegenwärtig von den großen Gesellschaften gehaltenen langfristigen Konzessionen. Die Regierung würde neue Konzessionen für einen Zeitraum von jeweils 25 Jahren erteilen. Zur Bewältigung der neuen Aufgaben wurde ein neues Ministerium geschaffen, das Ministerium für Staatsbeteiligung, dessen Leitung Präsident Kaunda sich selbst vorbehielt. Privatrechtlicher Arm dieses Ministeriums wurde die Zambian Industrial and Mining Corporation (ZIMCO), die gleichzeitig Holdinggesellschaft für die Industrial Development Corporation (INDECO) und die gleichzeitig neugegründete Mining Development Corporation (MINDECO) wurde.

Der Verstaatlichungsvorgang vollzog sich in ähnlicher Form wie in Chile. Zunächst wurden die beiden Konzerne insofern entflochten, als alle Betriebe und Vermögenswerte, die nicht mit der Förderung und Verarbeitung sowie mit dem Absatz von Kupfer zusammenhingen, abgesondert wurden. Dieses Sondervermögen wurde der von Präsident Kaunda gewünschten Verstaatlichung nicht unterworfen. Sodann wurde das Reinvermögen der Gesellschaften am 31. Dezember 1969 ermittelt. Es belief sich auf $\$ 576$ Millionen, so daß der vom Staat zu übernehmende 51 prozentige Kapitalanteil \$295 Millionen ausmachte. Für diesen Betrag gab die Zambia Industrial and Mining Development Corporation Limited (ZIMCO) auf US-Dollar lautende Schuldverschreibungen aus (loan stock, bonds), die von der zambischen Regierung garantiert wurden. Im Falle der Anglo-American Ltd.21 waren diese Schuldverschreibungen über einen Zeitraum von 12 Jahren, d. h. bis 1982, und im Falle des Roan Selection Trust Limited 22 über einen Zeitraum von acht Jahren, d. h. bis 1978, rückzahlbar. Der Zinssatz betrug wie in Chile 6 Prozent. Während der Laufzeit sollten die Abgabe- und Steuersätze für beide Gesellschaften nicht geändert werden. Sie betragen zur Zeit 73,05 Prozent des Gewinns. Die Úbernahme des Staatsanteils war außerden dadurch weiter kompliziert, daß die beiden Konzerne untereinander durch Beteiligungen der Anglo-American Ltd. an Gesellschaften des Roan Selection Trust verflochten waren und diese Beteiligung ebenfalls im Verhäitnis von 49:51 aufgeteilt werden mußten.

Der gesellschaftsrechtliche Aufbau der beiden Konzerne vor und nach der Verstaatlichung ist aus den nachfolgenden vier Darstellungen zu ersehen. Die verschiedenen, zu den beiden Konzernen gehörenden Grubengesellschaften wurden auf jeweils eine Gesellschaft verschmolzen, die Bancroft Mines Ltd. der Anglo-AmericanGruppe und die Mufulira Copper Mines Ltd. des Roan Selection Trust, deren

21 Explanatory Statement to the Members of Companies of the Anglo-American Group, Zambia, 27. 4. 1970, Lusaka, Zambia; Jahresbericht bis zum 30. 6. 1970, Zambia Copper Investments Ltd., Bermuda; Jahresbericht bis zum 25. 6. 1970, Zambia Anglo-American, Ltd., Bermuda.

22 Explanatory Statement for Meetings of Shareholders, 6. August 1970, Roan Selection Trust Ltd., Statement by the Chairman, Mr. A. S. Sardanis, Lusaka, Oct. 23, 1970, Lusaka, Zambia, Report to Shareholders, Roan Consolidated Mines Ltd., 30. Juni 1970. 
Namen in Nchanga Consolidated Copper Mines Ltd. (NCCM) und Roan Consolidated Mines Ltd. (RCM) geändert wurden. Jeweils 51 Prozent ihrer Anteile wurde auf die Mining Development Corporation (MINDECO) übertragen, die sogenannten "A“-Anteile zur Unterscheidung von den 49 Prozent im privaten Besitz verbleibenden „B“-Anteilen. Vor der Verstaatlichung des 51 prozentigen Anteils betrug die konsolidierte Beteiligung der Anglo-American Gruppe an der RST-Gruppe etwa 27 Prozent, so daß er nach Verstaatlichung etwa 12,25 Prozent ausmachte. Die als Gegenwert für die verstaatlichten Anteile ausgegebenen ZIMCO-Obligationen wurden ebenfalls dem Prozentsatz der ursprünglichen Beteiligung entsprechend zwischen der Anglo-American-Gruppe und dem Roan Selection Trust aufgeteilt.

Die Anteile an den der Verstaatlichung unterliegenden Grubengesellschaften der Anglo-American-Gruppe als auch deren abgesonderte Interessen wurden in eine in Bermuda gegründete Holding-Gesellschaft eingebracht, die Zambia Copper Investments Limited, an der zu 51,46 Prozent freie Aktionäre beteiligt sind. Der Anteil der Anglo-American Corporation von 48,54 Prozent wurde sodann auf eine ebenfalls in Bermuda neu gegründete Gesellschaft, die Zambian Anglo-American Limited, übertragen.

Im Bereich des Roan Selection T'rust Limited wurden zunächst die freien Aktionäre abgefunden, die bis dahin neben der American Metal Climax Corporation als Hauptaktionärin mit 57,7 Prozent an der Gesellschaft beteiligt gewesen waren, so daß die Gesellschaft danach hundertprozentig im Besitz der American Metal Climax lag. Sie fungierte sodann als Holding-Gesellschaft für den Anteil der American Metal Climax an der Roan Consolidated Mines Ltd. in Höhe von zwanzig Prozent.

Wie bei den Verstaatlichungen in Chile wurden auch in Zambia Betriebs- und Geschäftsvereinbarungen zwischen ZIMCO und der Anglo-American-Gruppe sowie der Roan-Selection-Gruppe abgeschlossen. Im Gegensatz zu den zwischen der chilenischen Regierung und der Anaconda Company geschlossenen Vereinbarung sieht der Verstaatlichungsvorgang in Sambia gegenwärtig noch nicht vor, daß auch die in privatem Besitz verbliebenen Anteile von 49 Prozent der Grubengesellschaften zu einem späteren Zeitpunkt - etwa in einer zweiten Phase - ebenfalls in den Besitz der sambischen Regierung übergehen sollen.

Im Rahmen eines Interviews im September 1969 sprach Kaunda von einem Geist des Nehmens und Gebens, von einer wirklichen Partnerschaft, von gegenseitigem Vorteil und von einem kontinuierlichen, nationalen Heilungsprozeß. Die Abkommen wurden am 24. Dezember 1969 unterzeichnet. Der sogenannte „Bancroft-Plan“ für die Anglo-American-Gruppe trat am 26. Juni 1970 und der „SubsidiariesPlan" des Roan Selection Trust am 1. 1. 1970 in Kraft — das Abkommen zwischen der chilenischen Regierung und Anaconda am 31. Dezember 1969. 


\section{Dezember 1969}

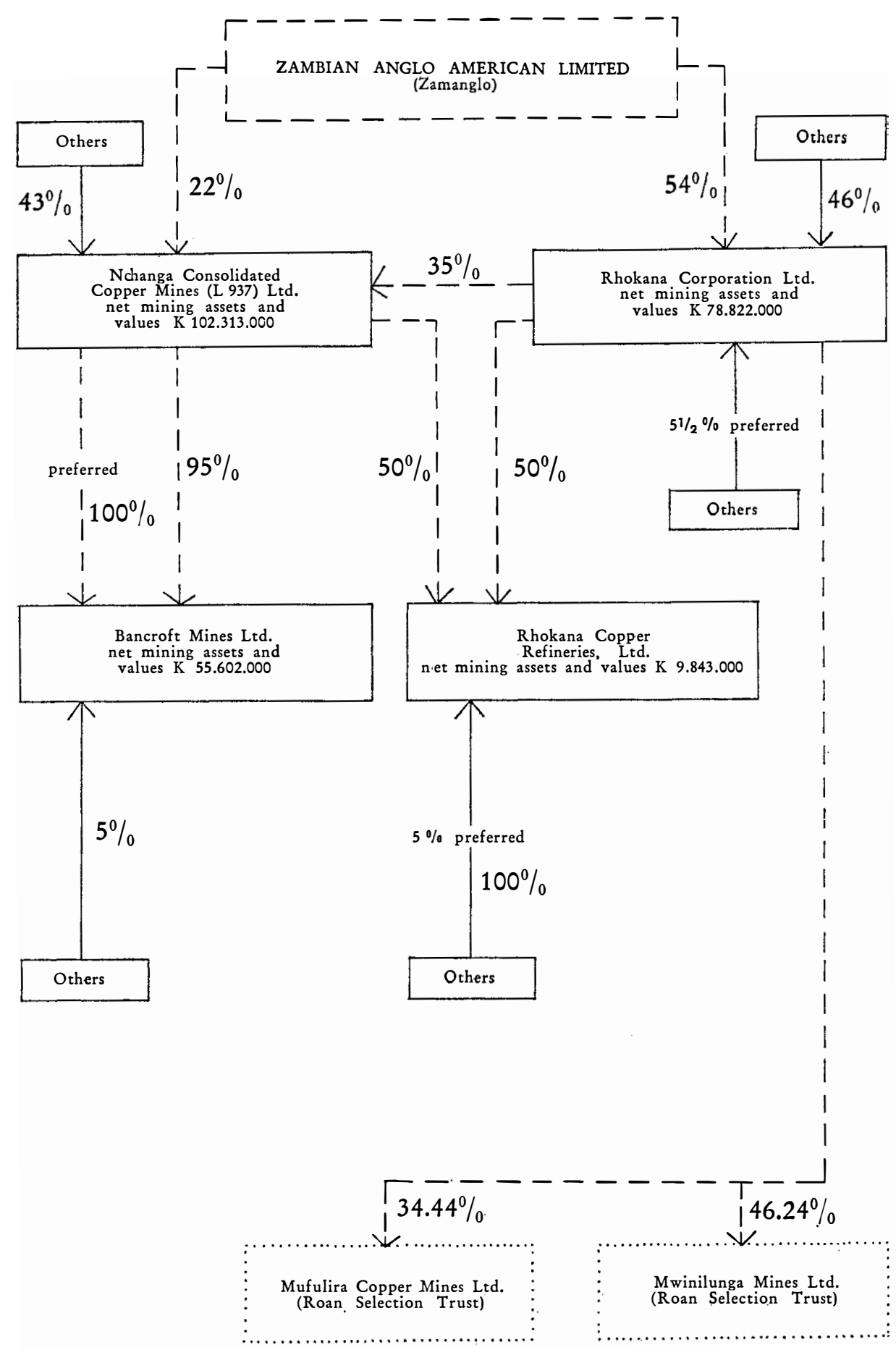




\section{Bancroft Scheme of Operations, 26 June 1970}

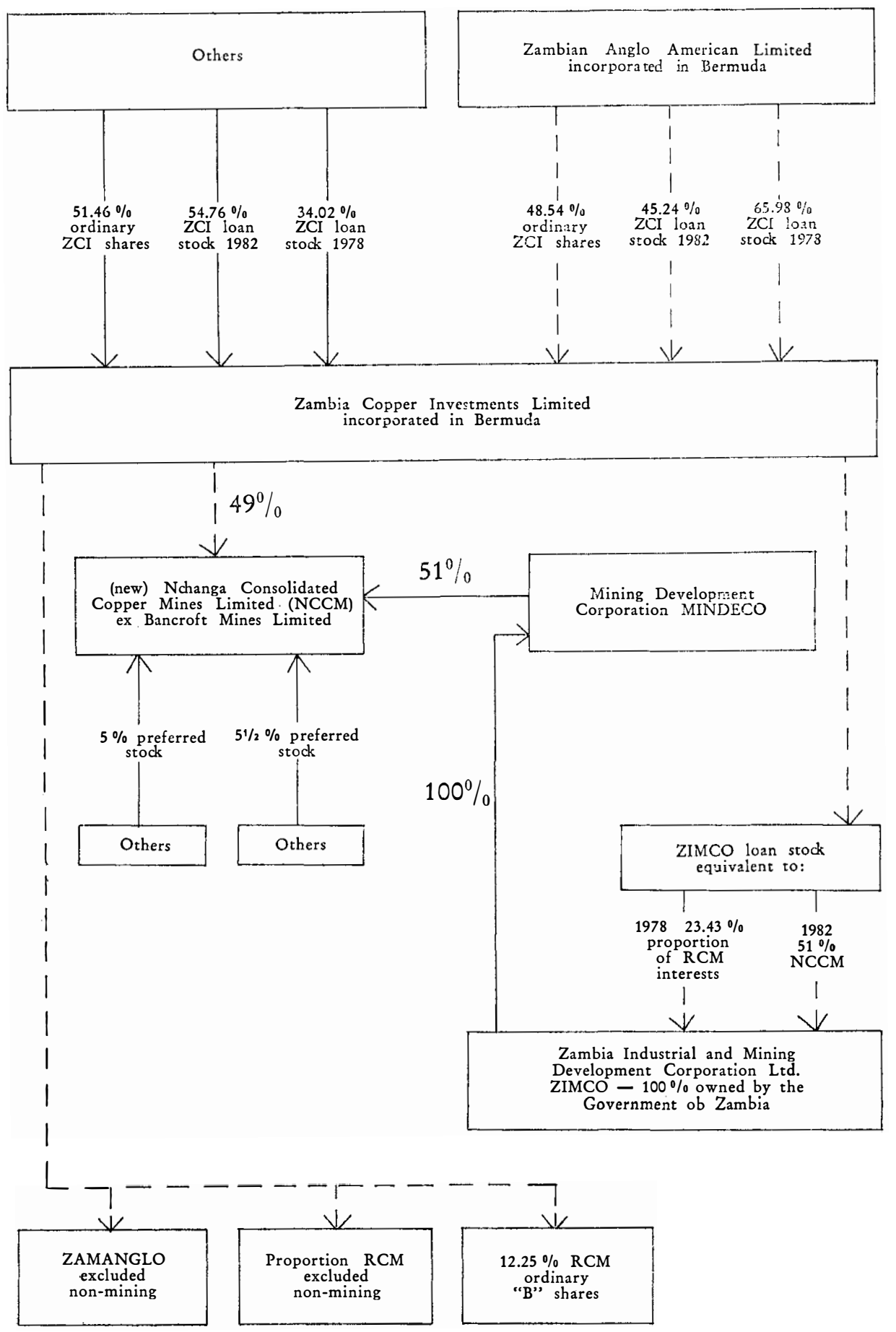




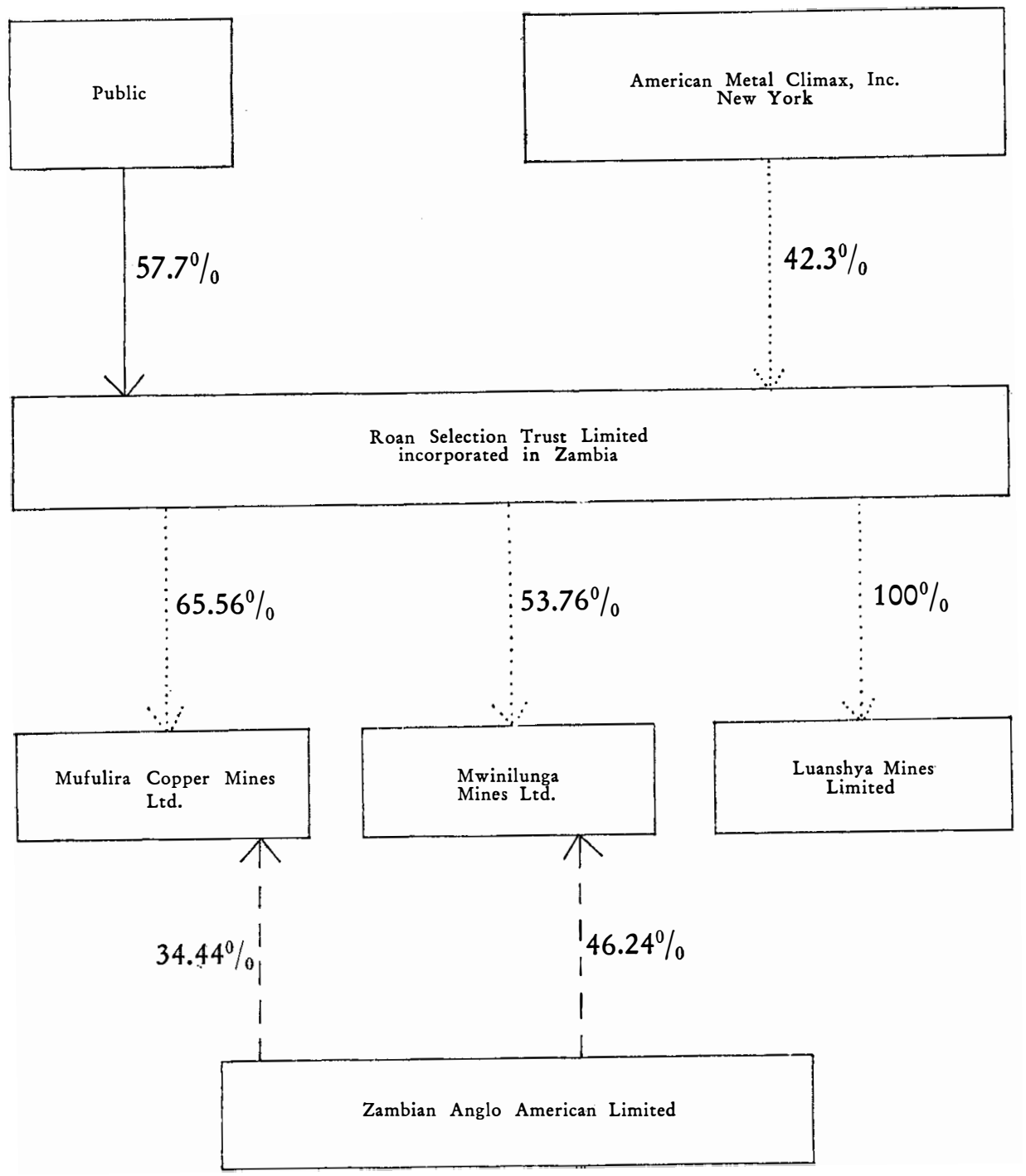

31. Dezember 1969 


\section{Subsidiaries Plan}

1 January 1970
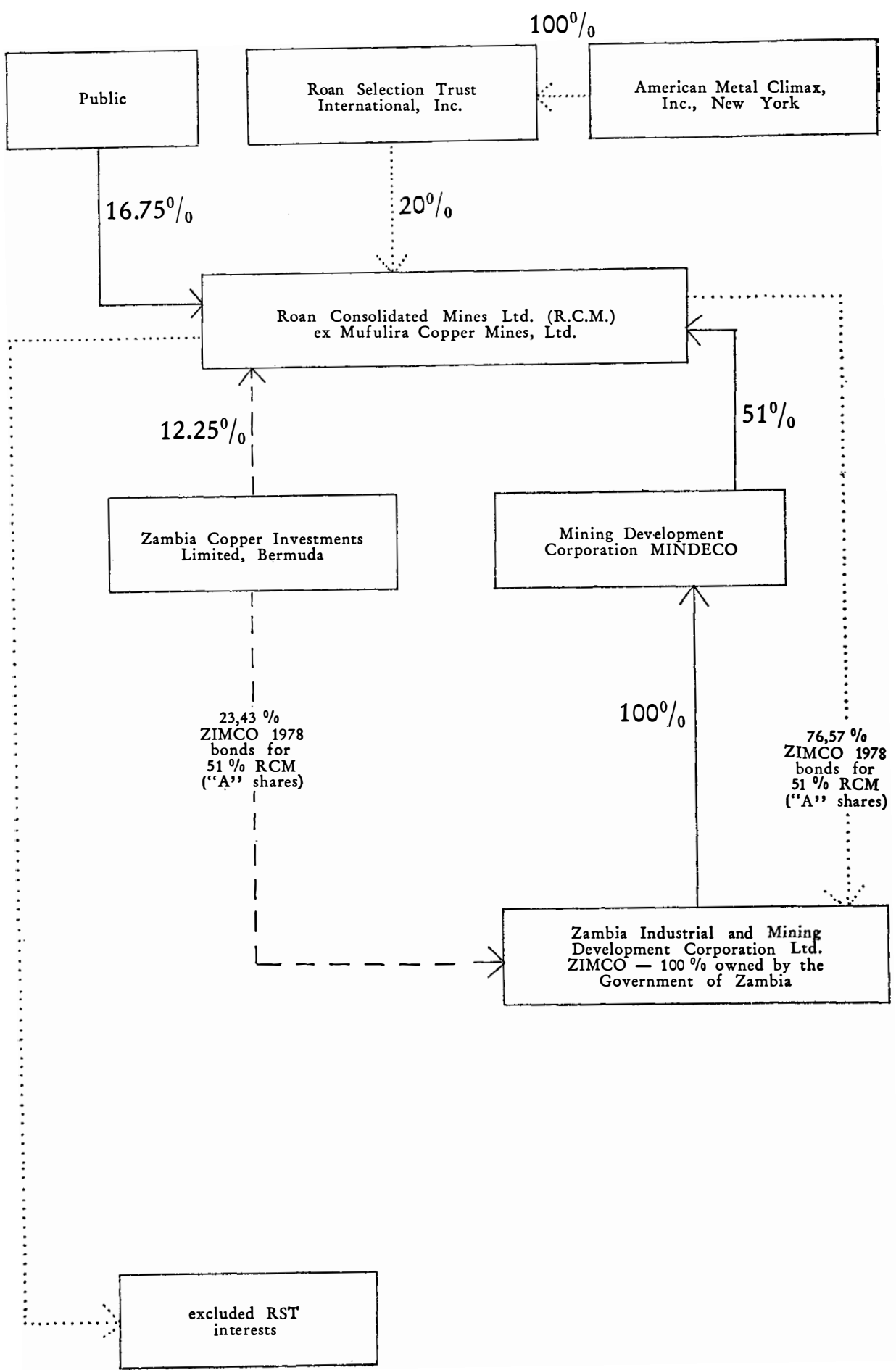


\section{Schlußwort}

In Chile hat man bei der schrittweisen vertraglichen Übernahme des Kupferbergbaus nicht haltgemacht. Schon zwei Monate nach Abschluß der Chilenisierungsabkommen des Präsidenten Frei, im August 1969, hatte der Ausschuß für Bergbauangelegenheiten des Kongresses mit den Stimmen der Linken bei Anwesenheit der rechtsorientierten Mitglieder des Ausschusses eine Gesetzesvorlage angenommen, die auf eine sofortige und völlige Verstaatlichung der Kupferbergwerksgesellschaften abzielte ${ }^{23}$. Als Präsident Frei die größeren Parteien zu einer Diskussion über die Verträge einlud, hatten die Linksparteien abgelehnt zu folgen, weil sie der Ansicht waren, daß die Verträge nationale Interessen verletzten ${ }^{24}$. Der neue Präsident Salvador Allende hatte schon im Zuge seines Wahlfeldzuges erklärt, daß er für entschädigungslose Enteignung der Kupferindustrie eintrete ${ }^{25}$. Von diesem Kurs ist er auch nach der Amtsübernahme nicht abgewichen ${ }^{26}$. Am 12. Juli billigte das chilenische Parlament eine Verfassungsänderung, die der Regierung das Recht der Kontrolle über die Bodenschätze übertrug. Am 15. Juli wurden die wichtigsten Kupferminen des Landes verstaatlicht. Eine Entschädigung ist zwar vorgesehen, deren Höhe ist jedoch noch ungewiß, da vom Buchwert der Unternehmen frühere übertriebene Gewinne und nicht bezahlte Steuern abgesetzt werden können ${ }^{27}$. Die jüngsten Entwicklungen in Chile könnten Auswirkungen auf Sambia haben, von dem man weiß, daß die Wirtschaftspolitik seines Präsidenten in bezug auf die Behandlung der Kupferindustrie jeweils den Ereignissen in Chile gefolgt ist. Es wäre durchaus denkbar, daß auch dort vorzeitig eine völlige Übernahme der Bergwerksgesellschaften durch den Staat angestrebt wird. 